\title{
Melanization and ageing are not drawbacks for successful agro- transformation of dark septate endophytes
}

\author{
Charlotte Berthelot ${ }^{1,2}$, Yoann Perrin ${ }^{1,2}$, Corinne Leyval ${ }^{1,2}$, Damien Blaudez ${ }^{1,2 \#}$ \\ ${ }^{1}$ Université de Lorraine, LIEC UMR 7360, Faculté des Sciences et Technologies, BP 70239, \\ F-54506 Vandoeuvre-lès-Nancy, France. \\ ${ }^{2}$ CNRS, LIEC UMR 7360, Faculté des Sciences et Technologies, BP 70239, F-54506 \\ Vandoeuvre-lès-Nancy, France. \\ \# Corresponding author: \\ Dr Damien Blaudez \\ damien.blaudez@univ-lorraine.fr
}

\begin{abstract}
Dark septate endophytes (DSEs) are melanin-enriched ascomycetous fungi that are abundant in stressed environments. However, little is known about their physiology and metabolism, and DSE genes have not been functionally characterized yet. Therefore developing molecular genetic tools to investigate the biological function of genes of interest in DSEs is of major significance. We investigated Agrobacterium tumefaciens-mediated transformation (ATMT) efficiency in eight DSE strains belonging to Cadophora sp., Cadophora malorum, Leptodontidium sp., Phialophora mustea, and Cladosporium cladosporioides. ATMT efficiency was DSE-dependent and ranged from $0.6 \%$ to $125 \%$. The transformants were mitotically stable and expressed the gfp marker. The patterns of transformant appearance as a function of time were diverse and also strain-dependent. We further focused on the effect of mycelium ageing on ATMT efficiency. Leptodontidium sp. Me07, Leptodontidium sp. Pr30, and C. cladosporioides CBS 101367 were significantly more transformed using fifteen-day-old mycelium $(44.5,6.9$, and $1.1 \%$, respectively) as compared to two-day-old mycelium $(121,28.7$, and 25.1, respectively), whereas P. mustea Pr29 was more transformed using young mycelium (21.5\% compared to $5.3 \%$ for the old mycelium). Finally, we focused on the effect of melanin content on ATMT efficiency. Melanin content in mycelium ranged from 0.9 to $3.2 \mathrm{mg}$ per gram of dry weight. Tricyclazole, an inhibitor of melanin biosynthesis, negatively modulated melanin content, while copper, an inducer of melanin biosynthesis, positively modulated them. However there was no correlation between hyphal melanin content and ATMT efficiency.
\end{abstract}

Keywords

Cadophora; Cladosporium; endophytic fungi; Leptodontidium; melanin; Phialophora mustea 


\section{Introduction}

Dark septate endophytes (DSEs) are miscellaneous, ascomycetous fungi hosted by plant roots. They are distributed worldwide, under various climates ranging from polar to tropical ecosystems (Mandyam \& Jumpponen, 2005). They are common under harsh conditions such as arid, dry, saline, and polluted environments (Knapp et al. 2012; Vrålstad et al. 2002; Zhang et al. 2013). Their tolerance to these unfavorable conditions may be partially explained by the structure of their hyphae which possess melanin-enriched cell walls (Ban et al. 2012). The relatively high abundance of DSEs in stressed habitats suggests that they might have an important function for host survival in these ecosystems (Likar \& Regvar, 2013). However, their effects on plant growth are still poorly elucidated. Several studies and meta-analyses support the view that DSEs could have a beneficial role on plant growth and fitness (Addy et al. 2005; Alberton et al. 2009; Mandyam \& Jumpponen, 2015; Mayerhofer et al. 2013; Newsham 2011). Several studies report plant-growth-promoting abilities of DSEs through increased biomass production and protection against biotic (pathogen) (Khastini et al. 2014; Tellenbach et al. 2013) or abiotic stress (drought, salinity, pollution) (Barrow 2003; Deram et al. 2011; Likar \& Regvar, 2013; Sonjak et al. 2009). Although DSEs might be an important component of plant tolerance, little is known about the molecular mechanisms that underpin their interactions with host plants.

The sequencing of the genomes of Cadophora sp., Leptodontidium sp., Microdochium bolleyi, Periconia macrospinosa, and Harpophora oryzae (David et al. 2016; Xu et al. 2014) was completed recently. However, DSE genes/proteins have not been functionally characterized yet. Therefore developing molecular genetic tools to investigate the biological function of genes of interest in DSEs is of major significance. The generation and characterization of knock-out mutants represent a powerful strategy for deciphering the roles of these genes/proteins (Herrera-estrella et al. 2004; Jiang et al. 2013). Among the techniques used for fungal transformation (particle bombardment, electroporation, PEG transformation...), Agrobacterium tumefaciens-mediated transformation (ATMT) is considered as the most promising one (Mullins \& Kang, 2001). A number of fungi such as yeasts, or pathogenic, mycorrhizal, endophytic and biocontrol fungi are indeed amenable to this transformation system (Michielse et al. 2005). Additionally, ATMT has several advantages. First, it does not require protoplasts or biolistic methods to transform cells and can be performed directly from mycelium. Second, it can generate a high percentage of transformants with a single-copy and randomly integrated DNA to facilitate the isolation of 
tagged genes. Finally, its application has been widely used for studies involving gene knockout, over-expression, complementation, and generating random integrations (Meyer 2008; Michielse et al. 2005).

Up to now, ATMT of DSE has only been performed on the four species Cladosporium cladosporioides, Harpophora oryzae, Cadophora finlandica and Phialocephala fortinii (Gorfer et al. 2007; Su et al. 2013; Zhang et al. 2011), and more investigations are required to better understand the parameters that influence their transformation. ATMT protocols for various fungi indicate that a number of parameters affect transformation efficiency. These parameters include (i) fungal parameters such as the choice of the species and the initial material (e.g. spores, protoplasts, mycelium) (Michielse et al. 2005), (ii) bacterial parameters such as the A.tumefaciens strain and its cell density (Flowers \& Vaillancourt, 2005; Tzima et al. 2014), and (iii) co-cultivation conditions such as the use of acetosyringone, incubation time, and temperature (Combier et al. 2003; Tzima et al. 2014). Among these factors, the addition of acetosyringone in the culture medium and the incubation time of the fungalbacterial co-cultivation are usually reported as important drivers of a successful ATMT. By contrast, the effect of mycelium ageing has been poorly investigated. Moreover, the effect of hyphal melanin content on ATMT efficiency has not been studied yet. Melanin is a dark pigment that provides structural rigidity to cell walls and protects cells against abiotic (UV, drought, metal) and biotic stress (lytic enzymes, pathogen attacks, antibiotics, drugs) (Butler \& Day, 1998; Van Duin et al. 2002; Gessler et al. 2014). According to Zhan et al. (2011) and Wheeler (1983), DHN-melanin, synthesized from 1,3,6,8-tetrahydroxynaphtalene, is admittedly the main form of melanin produced by ascomycetes, and tricyclazole was reported as an inhibitor of DHN-melanin synthesis. Conversely, copper treatment promoted melanin synthesis in cell walls of several fungi such as Amorphotheca resinae, Aureobasidium pullulans, and Gaeumannomyces graminis (Fogarty \& Tobin, 1996; Gadd \& Griffiths, 1980) DSEs are therefore suitable melanin-enriched fungal models to test whether a high melanin content in cell walls can affect ATMT. Finally, the majority of studies have investigated the transformation of a single strain or species using different protocols, so that generalizing the data is rather tricky.

We compared ATMT efficiency among eight strains belonging to four different DSE genera with an emphasis on the effect of (i) mycelium ageing, and (ii) the mycelium melanin content on ATMT efficiency. 


\section{Materials and methods}

\subsection{Strains, plasmid, and growth conditions}

Eight DSEs were used. The species, their origin and accession numbers are detailed in Table 1. DSEs were cultured on malt extract agar (MEA) medium at $24^{\circ} \mathrm{C}$ in the dark. The Agrobacterium tumefaciens strain EHA101 was used for fungal transformation. It harbors the pFAT-gfp binary vector that carries (i) the bacterial hygromycin B phosphotransferase ( $h p h)$ ORF under the transcriptional control of the Aspergillus nidulans $\operatorname{trp} C$ promoter, and (ii) the green fluorescent protein ( $g f p$ ) ORF under the control of the $A$. nidulans glyceraldehyde-3phosphate promoter (Fitzgerald et al. 2003). The bacteria were grown at $28^{\circ} \mathrm{C}$ on LuriaBertani (LB) agar medium supplemented with $100 \mu \mathrm{g} \mathrm{ml}^{-1}$ spectinomycin.

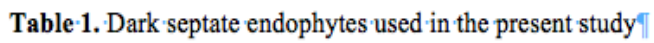

\begin{tabular}{|c|c|c|c|c|}
\hline Strains $\bar{\square}$ & Taxonomic affiliation & Acc. number & Origin & Colony color (Munsell chart) \\
\hline DSE 1049 & Cadophora $\mathrm{sp}$ & JN859258 & Vági et al.2014 & $10 \mathrm{YR}(3 / 2)$ Very dark greenish brown \\
\hline CBS 100584 & Cadophora malorum & AY249062 & $\mathrm{CBS}^{1}$ & 10YR(2/1) Black \\
\hline $\mathrm{Va} 47$ & Cladosporium cladosporioides & KX22756 & This study & 10YR(4/2) Dark grayish brown \\
\hline CBS $101367 \square$ & Cladosporium cladosporioides & HM148002 & $\mathrm{CBS}^{1}$ & $5 \mathrm{Y}(4 / 4)$ Olive \\
\hline $\mathrm{Me} 07$ 마 & Leptodontidium $\mathrm{sp}$ & KU886584 & Berthelot et al. 2016 & $5 Y R(5 / 2)$ Reddish gray \\
\hline $\operatorname{Pr} 30$ & Leptodontidium $\mathrm{sp}$ & KU886583 & Berthelot et al.2016 & $10 \mathrm{YR}(6 / 1)$ Gray \\
\hline Va46 & Phialophoramustea & KX622757 & This study & $10 \mathrm{R}(5 / 8) \operatorname{Red}$ \\
\hline $\operatorname{Pr} 29$ & Phialophora mustea & KU886582 & Berthelot et al.2016 & 7.5YR(6/6) Reddish brown \\
\hline
\end{tabular}

'CBS: Centraalbureau voor Schimmelcultures, The Netherlands. ๆ

\subsection{Molecular identification of the fungal species}

Fungal DNA was extracted using a REDextract-N-AmpTM Plant PCR kit (SigmaAldrich, St-Quentin-Fallavier, France) according to the manufacturer's protocol. The internal transcribed spacer (ITS) region was amplified using the primers ITS1 and ITS4 (White et al. 1990). Twenty microliters of a mixture containing $0.2 \mu \mathrm{M}$ of each primer, $4 \mu \mathrm{l}$ of genomic DNA, $5.2 \mu \mathrm{l}$ of water and $10 \mu \mathrm{l}$ of REDExtract-N-Amp PCR ready mix were used for PCR amplification. The following PCR program was used: an initial denaturation step of 3 min at $94^{\circ} \mathrm{C}$, followed by 40 cycles of $94^{\circ} \mathrm{C}$ for $1 \mathrm{~min}, 55^{\circ} \mathrm{C}$ for $1 \mathrm{~min}$, and $72^{\circ} \mathrm{C}$ for $1 \mathrm{~min}$, and a final extension step at $72^{\circ} \mathrm{C}$ for $10 \mathrm{~min}$. PCR products were cloned into the pGEM-T ${ }^{\circledR}$ vector system I (Promega, Charbonnières-les-Bains, France), and the corresponding plasmids were sequenced. The ITS sequences were used to retrieve similar sequences from GenBank using the NCBI BLAST program. Sequence alignment was carried out using Clustal X. Bootstrap 
analysis was performed with 1,000 replications. The neighbor-joining (NJ) tree was drawn with the $\mathrm{p}$ differences model and gamma for rate heterogeneity using MEGA 6 (Tamura et al. 2013).

\subsection{A. tumefaciens-mediated transformation}

DSEs were transformed by $A$. tumefaciens following a modified version of the protocol used by Pardo et al. (2002). Electro-competent cells of A. tumefaciens strain EHA101 were transformed with the pFAT-gfp vector, using a Cell-Porator apparatus (Invitrogen, Cergy Pontoise, France). The transformants were selected on LB medium containing $100 \mu \mathrm{g} \mathrm{ml}^{-1}$ of spectinomycin. A. tumefaciens was grown overnight at $150 \mathrm{rpm}$ in LB medium containing $100 \mu \mathrm{g} \mathrm{ml}^{-1}$ spectinomycin. The cells were subsequently diluted to an $\mathrm{OD}_{600}$ of 0.2 in LB induction medium (IM) containing $200 \mu \mathrm{M}$ acetosyringone and $100 \mu \mathrm{g} \mathrm{ml}^{-1}$ of spectinomycin, and further incubated until an $\mathrm{OD}_{600}$ of 0.6 was reached. In a first experiment, and in order to compare the effect of ageing of the initial mycelium (used for the pregrowth step) on ATMT efficiency, fungal plugs were taken from two contrasting zones of the fungal colonies (grown on MEA medium). The 2-day-old mycelium corresponded to fresh mycelium at the close periphery of the mother colony, whereas the 15-day-old mycelium was taken from the deep center of the colony. In a second experiment, to study the impact of melanin content of mycelia on ATMT efficiency, fungal plugs were taken from fungal cultures growing on MEA medium or on the same medium amended with copper $(200 \mu \mathrm{M})$ or tricyclazole $\left(10 \mu \mathrm{g} \mathrm{ml}^{-1}\right)$. In both experiments, 1 -mm agar plugs ( 20 plugs/Petri dish) were deposited on sterilized cellophane membranes on MEA medium (first experiment) or on the same medium amended or not with copper $(200 \mu \mathrm{M})$ or tricyclazole $\left(10 \mu \mathrm{g} \mathrm{ml}^{-1}\right)$ (second experiment). After three days of fungal pre-growth, co-cultivation between the fungal colonies and A.tumefaciens was done as described by Bellion et al. (2007). The media used during the co-cultivation step were the same than the ones used in the pre-growth step. Finally, the fungal colonies, together with the cellophanes membranes, were transferred to MEA medium containing $250 \mu \mathrm{g} \mathrm{ml}^{-1}$ hygromycin B (selection step). The appearance of the transformants was recorded every week. Resistant colonies were individually harvested and isolated for vegetative propagation on the same selective medium.

\subsection{Mitotic stability}

The mitotic stability of the transformants was determined by analyzing the stability of hygromycin B resistance. Five randomly selected transformants per DSE were sub-cultured every three weeks alternatively on MEA medium with or without hygromycin B. 
Transformants were classified as stable when they remained resistant to hygromycin B after seven sub-cultures.

\subsection{Molecular analysis of the transformants}

To confirm the positive transformants for T-DNA insertions in the host genomes, five transformants per DSE were randomly selected to amplify two inserted sequences. The $h p h$ gene was amplified with the primers hphF (5'-ATGCCTGAACTCACCGCGACGTCTGT-3') and hphR (5'-CTATTCCTTTGCCCTCGGACGAGT-3'), and the $g f p$ gene was amplified with the primers gfpF (5'-ATGAGCAAGGGCGAGGAACTGTTC-3') and gfpR (5'TCACTTGTACAGCTCGTCCATG-3'). Fungal DNA was extracted from the mycelia using the REDextract-N-Amp ${ }^{\text {TM }}$ Plant PCR kit (Sigma-Aldrich) according to the manufacturer's protocol. PCR amplification was performed using Phire Hot Start II DNA polymerase (Fischer Scientific, Illkirch, France) with the following program: initial denaturation at $98^{\circ} \mathrm{C}$ for $30 \mathrm{~s}, 40$ cycles of $98^{\circ} \mathrm{C}$ for $5 \mathrm{~s}, 63^{\circ} \mathrm{C}$ for $5 \mathrm{~s}$ and $72^{\circ} \mathrm{C}$ for $10 \mathrm{~s}$, and a final extension step at $72^{\circ} \mathrm{C}$ for $1 \mathrm{~min}$. To verify the absence of contaminating A. tumefaciens cells within the resistant fungal colonies, a control PCR was also performed with the primers SpecF (5'ATGAGGGAAGCGGTGATCGCCGAA-3') and SpecR (5'TTATTTGCCGACTACCTTGGTGAT-3') that are specific to the spectinomycin resistance gene located on the pFAT-gfp plasmid but outside the T-DNA region. PCR conditions were the same as described above except that the annealing temperature was set to $51^{\circ} \mathrm{C}$. Negative controls were obtained from the wild-type fungal strains whereas A. tumefaciens was used as a positive control. The PCR products were analyzed by electrophoresis on $1 \%$ agarose gels.

\subsection{Fluorescence microscopy}

To screen for GFP expression in the transformants, three randomly chosen transformants per DSE were cultured for one week on a glass slide covered by a thin layer of MEA medium. A filter was used to select an excitation wavelength within the $475-500 \mathrm{~nm}$ band, and fluorescence emission was recorded above $520 \mathrm{~nm}$. The wild-type strains of the different DSE species were used as controls.

\subsection{Melanin extraction and quantification}

Melanin extraction was performed from 15 day-old colonies grown on MEA plates supplemented with or without $200 \mu \mathrm{M} \mathrm{CuCl}_{2}$ or $10 \mu \mathrm{g} \mathrm{ml}^{-1}$ tricyclazole. Mycelia were harvested and dried overnight at $50^{\circ} \mathrm{C}$ and ground to a thin powder. The extraction process was carried out according to the method of Zou et al. (2010), with modifications. The powder (1g) was washed for 5 min with $30 \mathrm{ml}$ of distilled water, followed by a centrifugation step at 
4,000 rpm for $5 \mathrm{~min}$. The supernatant was discarded, and the pellet was resuspended in $\mathrm{NaOH}$ $6 \mathrm{M}$ to reach a final $\mathrm{pH}$ of 12 . The mixture was sonicated at $40 \mathrm{~Hz}$ for $24 \mathrm{~h}$ at $50^{\circ} \mathrm{C}$ and centrifuged at 4,000 rpm for $5 \mathrm{~min}$. The alkaline pigmented extract was then acidified to $\mathrm{pH} 2$ with $\mathrm{HCl} 8 \mathrm{M}$ to precipitate melanin, followed by a centrifugation step at 10,000 rpm for 20 min. Melanin was finally washed with chloroform and ethyl acetate. For quantification, melanin was dissolved in $\mathrm{NaOH} 0.1 \mathrm{M}$ and the $\mathrm{OD}_{400 \mathrm{~nm}}$ of the solution was determined. Blanks were performed with $\mathrm{NaOH} 0.1 \mathrm{M}$. A standard curve was obtained with synthetic melanin (M8631, Sigma-Aldrich).

\subsection{Data analysis}

Statistical analyses were performed with R 3.1.3 (R Core Team, 2013) software. Normality of the data was tested using a Shapiro-Wilk test. Data were then analyzed by oneway ANOVA or by a one-way non-parametric Kruskal-Wallis test (alpha level of 0.05).

Furthermore, analysis of covariance (ANCOVA) was used to study the optimal time to obtain transformants for the different DSEs. The effect of compounds reducing or stimulating melanin synthesis, mycelium ageing and the interaction between these two factors on ATMT of the DSEs was tested by a two-way ANOVA. Pearson correlation coefficients (r) were calculated to determine the relationship between mycelial melanin content and ATMT efficiency. All values are means \pm standard errors (SE). Percentage values were arcsintransformed before statistical analyses.

\section{Results}

\subsection{Molecular identification of the fungal species}

Eight DSEs were used (Fig. 1, Table 1). The isolation and the taxonomic affiliation of Phialophora mustea Pr29, Leptodontidium sp. Pr30, and Leptodontidium sp. Me07 are reported in Berthelot et al. (2016). Cladosporium cladosporioides CBS 101367, Cadophora malorum CBS 100584, and Cadophora sp. DSE 1049 belong to fungal collections. Va47, and Va46 were isolated in the present study from birch roots, and their taxonomic affiliations were performed by analyzing their ITS1-5.8S-ITS2 regions. A NJ phylogenetic tree was drawn using sequences from DSEs as well as from other endophytic fungi (Table S1). Va46 had a very close evolutionary relationship to Phialophora mustea (JN123359) and to the previously described P. mustea Pr29 (Fig. 1). Va47 had a close evolutionary relationship to Cladosporium cladosporioides. Additionally, a phylogenetic analysis relying on the maximum likelihood method was also performed and confirmed the confidence level of the 
clustering (data not shown). Sequence data were deposited in GenBank under the following accession numbers: KX622757 (C. cladosporioides Va47), and KX622756 (P. mustea Va46). As previously reported for several fungal species (Cardoso et al. 2007; Vellinga 2006), mycelium color was described (Table 1) using the Munsell chart (Munsell, 1954). DSEs clustered into four groups (Table 1): (i) the yellow red (YR) group (Cadophora sp. DSE 1049, C. malorum CBS 100584, C. cladosporioides Va47, Leptodontidium sp. Pr30, Leptondontidium sp. Me07, P. mustea Pr29), (ii) the red (R) group (P. mustea Va46), and (iii) the olive group (C. cladosporioides CBS 101367).

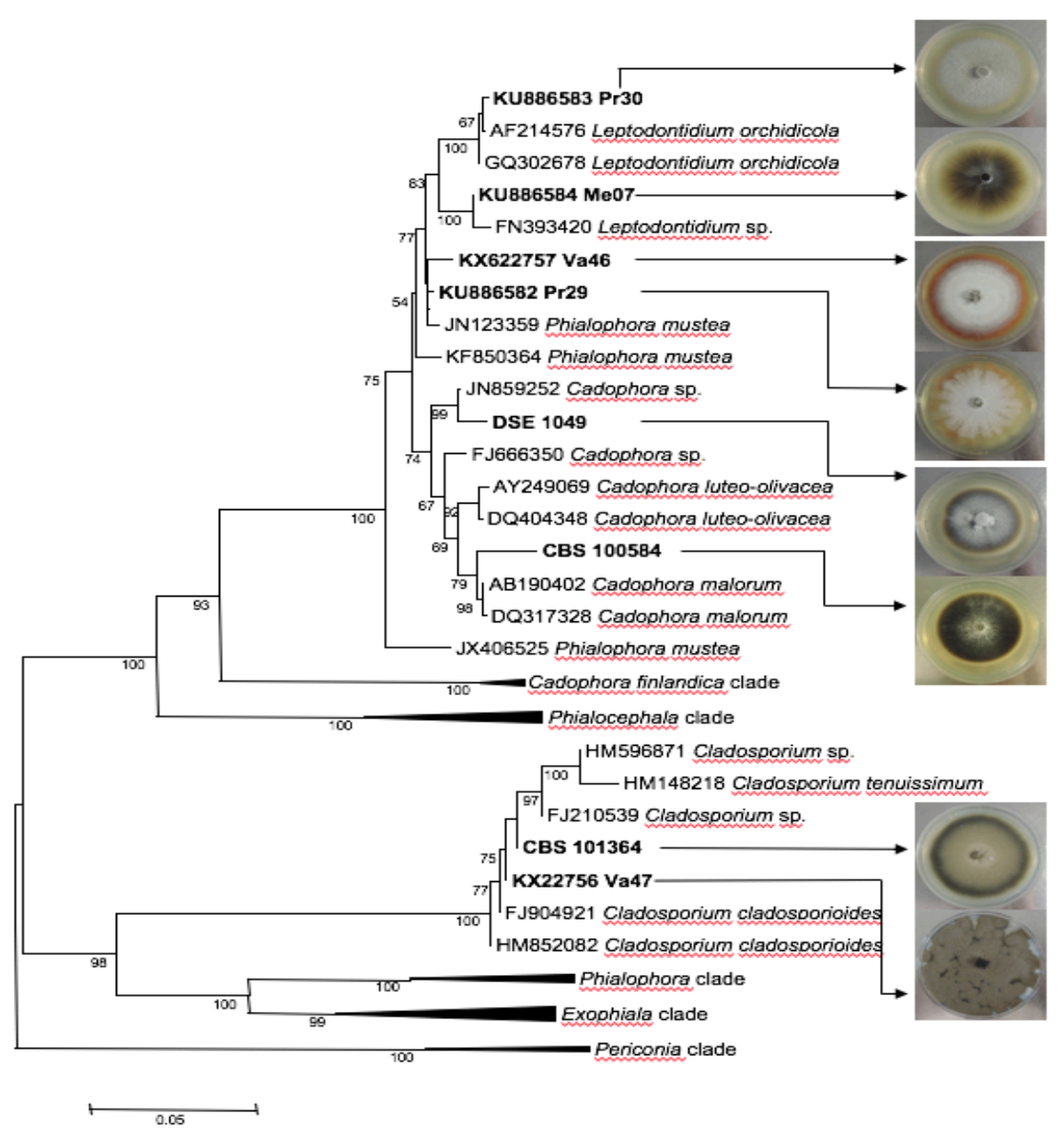

Fig. 1. Neighbor-Joining phylogram showing the phylogenetic relationships between the species based on ITS15.8S-ITS2 sequences and colonies of the DSEs used in the present study.

Bootstrap values greater than $50 \%$ are indicated along nodes. The evolutionary history was inferred using the Neighbor-Joining method (Saitou and Nei 1987). Evolutionary analyses were conducted in MEGA6 (Tamura et al. 2013). The DSEs used in the present study (in bold) were compared with known and selected fungal species from GenBank with high sequence similarities. Five clades were compressed for the sake of clarity. The corresponding sequences are provided in Table S1. Pictures were taken after 14 days of growth on MEA medium. 

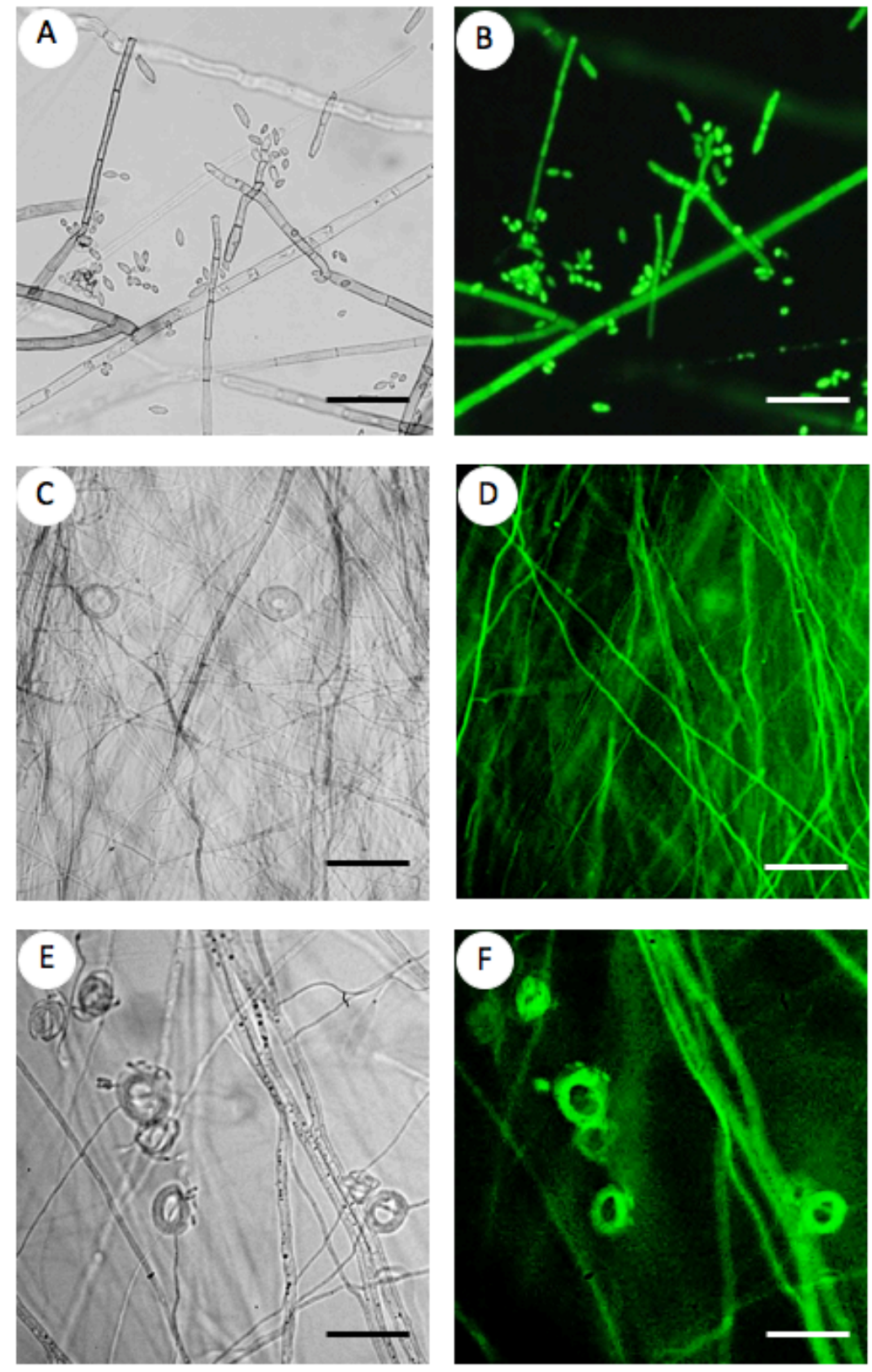

Fig. 2. Fluorescence microscopy analysis of the selected DSE transformants.

A-B: Cladosporium cladosporioides CBS 101367; C-D: Cadophora sp. DSE 1049, E-F: Phialophora mustea Pr29. Images from the same hyphae and spores are shown in bright field (A-C-E) and GFP fluorescence (B-DF). Pictures were taken after two weeks of growth on MEA medium. Bars $=50 \mu \mathrm{m}$.

\subsection{Confirmation of DSE ATMT}

To establish the mitotic stability of ATMT, five randomly selected transformants per DSE (when available) were grown for seven consecutive cultures with or without hygromycin B. All the transformants were resistant to hygromycin B (data not shown), confirming a stable 
maintenance of the $h p h$ marker gene across the successive generations. The stability of the transformants was also confirmed by checking for the presence of the $h p h$ and $g f p$ genes by PCR. The expected 1,020 bp $(h p h)$ and $717 \mathrm{bp}(g f p)$ fragments were detected in all transformants, but not in the wild type strains (data not shown). Conversely, there was no amplification of the spectinomycin resistance gene. This result therefore confirms T-DNA integration into the DSE genomes and also rules out any remaining bacterial contamination in mycelium. Moreover, GFP-derived fluorescence was recorded in hyphae and conidia (Fig. 2).

Untransformed strains did not emit any GFP-derived fluorescence under the same excitation and emission conditions. However, all transformants emitted GFP-derived fluorescence, and fluorescence intensity was transformant-dependent. We also noted that fluorescence intensity was attenuated by the dark pigmentation of several DSE species. Cadophora sp. DSE 1049 particularly emitted a weak GFP signal (data not shown).

\subsection{Comparison of ATMT efficiency among the different DSE species}

To compare ATMT efficiency between DSEs, each of the eight strains belonging to four different genera was co-cultivated with Agrobacterium and we selected fungal transformants. Statistical analyses showed that ATMT efficiency was strain-dependent $(\mathrm{P}<$ $0.001)$.

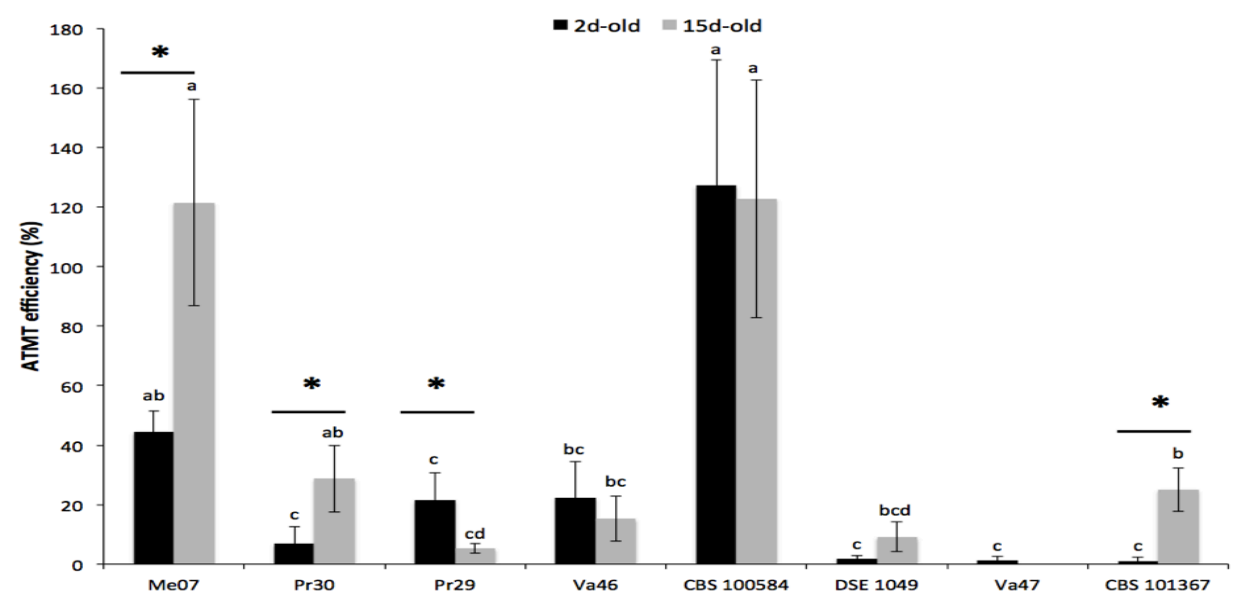

Fig. 3. Transformation efficiency of eight DSEs.

Data represent the means $( \pm \mathrm{SE} ; \mathrm{n}=10)$ of the ATMT efficiency levels for the different DSEs. Black and grey bars represent $2 \mathrm{~d}$-old and $15 \mathrm{~d}$-old mycelium respectively. The different letters above the bars indicate significant differences between DSEs whereas asterisks indicate significant differences between ages of mycelium (Kruskal-Wallis, $\mathrm{P}<0.05$ ). 
Overall, ATMT efficiency ranged between 0.6 and 127\% (Fig. 3). C. malorum CBS 100584 and Leptodontidium sp. Me07 produced the highest number of transformants. For both DSEs, two independent transformants frequently originated from the same initial fungal plug. Compared to C. malorum CBS 100584 and Leptodontidium sp. Me07, the ATMT efficiency values for Leptodontidium sp. Pr30, P. mustea Pr29, P. mustea Va46, and C. cladosporioides CBS 101367 were significantly lower (between 12 and 18\%), and Cadophora sp. DSE 1049, and C. cladosporioides Va47 were poorly transformed (Fig. 3).

To compare the effect of ageing of the initial mycelium on ATMT efficiency, fungal plugs were taken from two contrasting zones of the fungal colonies corresponding to 2-and 15-day-old mycelia to prepare colonies to be used for co-cultivation (Fig. 3). The 2-day-old mycelium corresponded to fresh mycelium at the close periphery of the colony, whereas the 15-day-old mycelium was taken from the deep center of the colony. Contrasting color phenotypes were usually observed between the two zones (Fig. 1). Two-way ANOVA results revealed significant differences in ATMT efficiency among the DSEs and for the DSEmycelium ageing interaction, but there was no significant difference for the "mycelium age" parameter $(\mathrm{P}<0.01 ; \mathrm{P}<0.01$, and $\mathrm{P}=0.356$, respectively). However, ATMT efficiency of Leptodontidium sp. Me07, Leptodontidium sp. Pr30 and C. cladosporioides CBS 101367 was significantly higher with 15-d-old mycelium than with 2-d-old mycelium, while more transformants developed from P. mustea Pr29 2-d-old mycelium (Fig. 3). Then, we investigated whether 15-d-old and 2-d-old mycelia produced colonies with different hyphal densities during the pre-growth step. The number of hyphal tips were similar in both cases (data not shown). Finally, the efficiency of the transformation of C. malorum CBS 100584, C. cladosporioides Va47, Cadophora sp. DSE 1049, and P. mustea Va46 was not influenced by mycelium ageing (Fig. 3).

The first transformants were recorded after two weeks on the selective medium, whereas the last ones were recorded after eight weeks (Fig. 4). However, the pattern of appearance of the transformants as a function of time differed among the DSEs. Most of the C. malorum CBS 100584 and C. cladosporioides CBS 101367 transformants appeared during the second week, and the remaining transformants were recorded in the following two weeks. This was also the case for P. mustea $\operatorname{Pr} 29$ even if only $31.6 \%$ of the transformants appeared during week 2. Conversely, transformant appearance was more progressive for Leptodontidium sp. Me07, Leptodontidium sp. Pr30, and P. mustea Va46. Moreover, the 
delay to obtain the rare Cadophora sp. DSE 1049 transformants was longer: they only appeared during week 4.
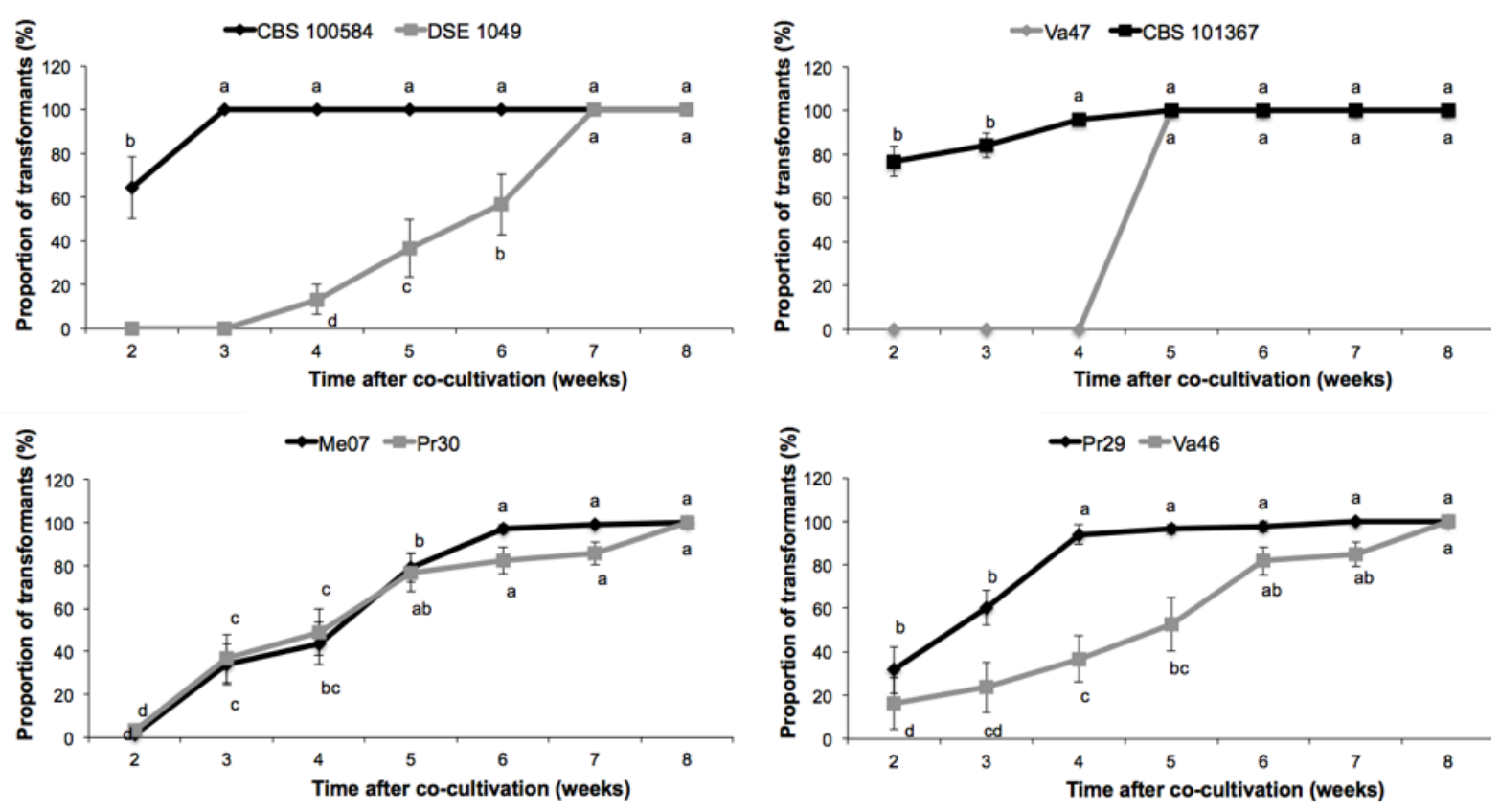

Fig. 4. DSE-dependent time course of the appearance of transformants on the selective medium.

The delay for transformant appearance was noted for the different strains of Cadophora (A), Leptodontidium (B), Cladosporium (C), and Phialophora (D). Data are given as means $( \pm \mathrm{SE} ; \mathrm{n}=10)$ of relative percentages of transformants detected at a given time. The total number of transformants for each strain was set to $100 \%$. Different letters above the bars indicate significant differences between weeks (ANCOVA, $\mathrm{P}<0.05$ ).

\subsection{Effect of melanin content on ATMT efficiency}

Melanin content was determined in the mycelium of the different strains. The extracted molecules were yellow to black, insoluble in water and in several organic solvents (chloroform, ethyl acetate, ethanol), soluble in $0.1 \mathrm{~N} \mathrm{NaOH}$, precipitated in $\mathrm{HCl}$ and were discolored by $\mathrm{H}_{2} \mathrm{O}_{2}$ (data not shown). Altogether these observations confirm that the extracted molecules possessed typical characteristics of melanin (Gessler et al. 2014). Among the eight strains of the five species, melanin content ranged from 0.9 to $3.2 \mathrm{mg} \mathrm{g}^{-1} \mathrm{DW}$ (data not shown). Pearson correlation coefficient was estimated between ATMT efficiency levels and melanin content, but there was no significant correlation $(\mathrm{P}=0.06, \mathrm{r}=-0.43)$.

To confirm this finding, we further evaluated the ATMT efficiency levels and the melanin content of four DSEs grown in the presence of a melanin synthesis inhibitor (tricyclazole) or a melanin synthesis inducer (copper) (Table 2). These DSEs were selected 
according to two criteria: their pigmentation phenotype (Table 1), and their different responses to transformation (recalcitrant to easily transformable). Under tricyclazole treatment, the colonies displayed lighter colors but also accumulated a red/brown pigment (Fig. 5). Under the copper treatment, they exhibited a darker aspect. Adding tricyclazole to the culture medium of Leptodontidium sp. Me07, Leptodontidium sp. Pr30, P. mustea Va46 and P. mustea Pr29 significantly decreased mycelial melanin content (Table 2). Conversely, adding copper increased the mycelial melanin content of the four DSEs. ATMT efficiency was only significantly reduced for Leptodontidium sp. Me07 under the copper treatment as compared to the control. Finally, the relationship between melanin content and ATMT efficiency was studied through Pearson correlation tests but no significant correlation was evidenced (Table 2).

Table 2. Relationship between melanin content and the transformation efficiency of four DSE

\begin{tabular}{|c|c|c|c|c|c|c|c|c|}
\hline \multirow[t]{2}{*}{ Strains } & \multicolumn{3}{|c|}{ Transformation efficiency (\%) } & \multicolumn{3}{|c|}{ Melanin content $\left(\mathrm{mg} \mathrm{g}^{-1}\right)$} & \multicolumn{2}{|c|}{ Pearson correlation } \\
\hline & Control & Copper & Tricyclazole & Control & Copper & Tricyclazole & P.value & $\mathrm{R}$ \\
\hline $\mathrm{Me} 07$ & $25.7 \pm 4.5^{\mathrm{a}}$ & $9.5 \pm 3.5^{\mathrm{b}}$ & $13.8 \pm 2.9^{\mathrm{ab}}$ & $0.9 \pm 0.0^{\mathrm{b}}$ & $1.3 \pm 0.1^{\mathrm{a}}$ & $0.5 \pm 0.1^{c}$ & 0.65 & -0.09 \\
\hline $\operatorname{Pr} 30$ & $12.4 \pm 3.8^{\mathrm{a}}$ & $6.7 \pm 5.1^{\mathrm{a}}$ & $13.8 \pm 5.5^{\mathrm{a}}$ & $1.5 \pm 0.1^{\mathrm{b}}$ & $3.9 \pm 0.8^{\mathrm{a}}$ & $0.8 \pm 0.1^{\mathrm{c}}$ & 0.42 & -0.17 \\
\hline $\operatorname{Pr} 29$ & $9.0 \pm 3.4^{\mathrm{a}}$ & $11.0 \pm 2.3^{\mathrm{a}}$ & $2.8 \pm 2.6^{\mathrm{a}}$ & $1.4 \pm 0.0^{\mathrm{b}}$ & $1.7 \pm 0.1^{\mathrm{a}}$ & $0.9 \pm 0.2^{\mathrm{c}}$ & 0.55 & 0.13 \\
\hline Va46 & $9.5 \pm 4.5^{\mathrm{a}}$ & $12.4 \pm 4.0^{\mathrm{a}}$ & $11.4 \pm 3.1^{\mathrm{a}}$ & $2.8 \pm 0.4^{b}$ & $7.6 \pm 1.0^{\mathrm{a}}$ & $0.5 \pm 0.0^{c}$ & 0.94 & 0.01 \\
\hline
\end{tabular}

Values are means $( \pm$ SE; $n=5)$. Within a given fungus, different letters indicate significant differences between treatments $(P<0.05$; KruskalWallis test).

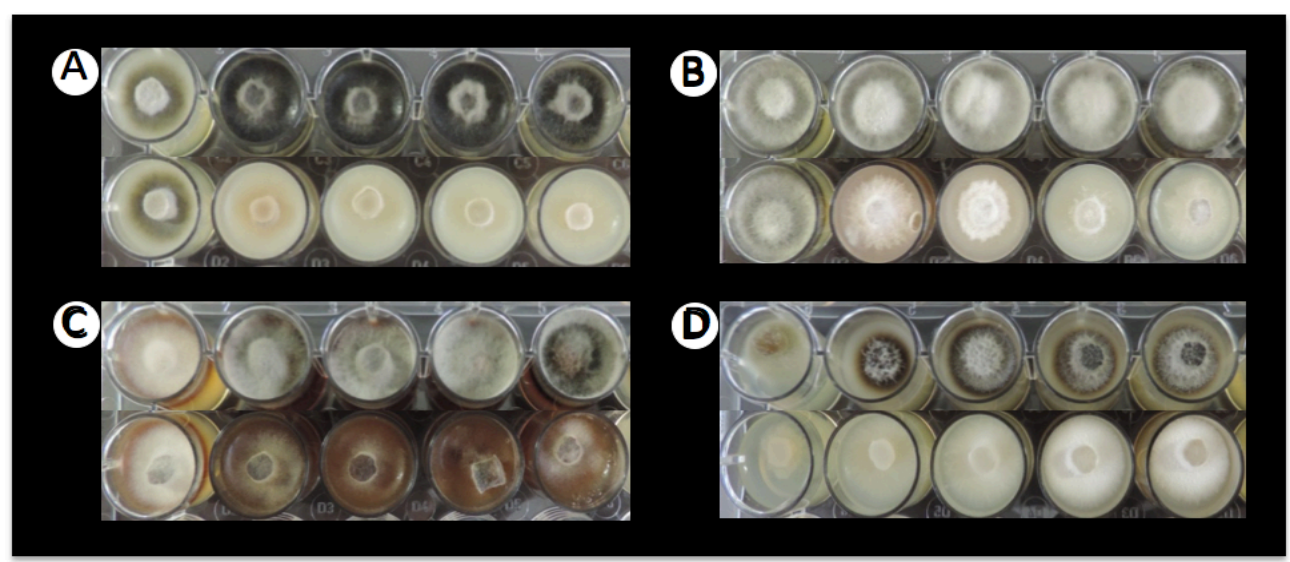

Fig. 5. Effects of copper and tricyclazole on the pigmentation of DSEs.

DSEs were grown on MEA medium amended with increasing concentrations of copper (upper rows) or tricyclazole (lower rows). Pictures were obtained after two weeks of growth. One representative picture out of three independent experiments is given for each strain/treatment combination. (A) Leptodontidium sp. Me07, (B) Phialophora mustea Pr29, (C) Phialophora mustea Va46, and (D) Leptodontidium sp. Pr30. From left to right, The $\mathrm{Cu}$ concentrations were: $0 ; 20 ; 100 ; 200 ; 500 \mu \mathrm{M}$ and the tricyclazole concentrations were: $0 ; 10 ; 20$; $30 ; 50 \mu \mathrm{g} / \mathrm{ml}$. 


\section{Discussion}

In the recent years, DSEs have been under focus because of their abundance in multiple ecosystems and the diversity of species and functions they offer (Jumpponen \& Trappe, 1998; Mandyam \& Jumpponen, 2014). They act as beneficial symbionts of a broad range of plants, so they are valuable in plant growth management (Newsham 2011; Upson et al. 2009; Usuki \& Narisawa, 2007). Despite the recent availability of DSE genomes (Cadophora sp., Leptodontidium sp. Harpophora oryzae, Microdochium bolleyi, Periconia macrospinosa...) (David et al. 2016; Xu et al. 2014) functional genomics studies involving DSEs are limited. Transcriptomic studies using micro-arrays or RNA-seq technologies have been performed to identify genes involved in the metal tolerance of Exophiala pisciphila and C. finlandica (Gorfer et al. 2009; Zhao et al. 2015). The only functional genomics data available from DSEs can be found in these two studies that report the functional characterization of a glutathione S-transferase and an Nramp metal transporter in E. pisciphila. Genetic transformation of DSEs represents an alternative and powerful tool to study gene functions in these organisms.

In the present study, we compare ATMT efficiency on a relatively large set of DSEs. We used eight strains belonging to four genera: Cadophora, Leptodontidium, Phialophora, and Cladosporium. To our knowledge, this is the first study that compares ATMT efficiency among DSE strains. We evidenced that ATMT efficiency was strain-dependent. We succeeded in transforming several Cadophora and Leptodontidium strains. The literature reports the successful transformation of four DSE species (Gorfer et al. 2007; Su et al. 2013; Zhang et al. 2011). Gorfer et al. (2007) report higher transformation rates (approximately $50 \%$ efficiency for $C$. finlandica) than we do. Transformation of C. cladosporioides has also been achieved (Zhang et al. 2011), but from spore materials, so that no comparison can be made with our study. Our results suggest that Leptodontidium sp. Me07 and C. malorum CBS 100584 were particularly sensitive to transformation. In both cases, several transformants often emerged from a single mother colony on the selective medium. Only very distinctive transformants (growing from opposite sides of the mother colony) were taken into account. Consequently, ATMT efficiency might have slightly been underestimated with these two DSEs. ATMT was also successful for the six other DSEs, even if transformation rates were rather low for Cadophora sp. DSE1049 and C. cladosporioides Va47. Specific optimization would be needed to efficiently perform ATMT with these particular strains. A large number 
of studies address this issue and reveal that specific parameters could influence ATMT performance. Among these, $\mathrm{pH}$, co-cultivation duration, temperature, the presence/absence of acetosyringone, the choice of binary vectors and bacterial strains are highly influential (Meng et al. 2007; Michielse et al. 2005; Wang et al. 2013; Xu et al. 2014). Based on a literature review of ATMT of ascomycetes and the growth requirements of the DSE genera we investigated, we selected a few standard conditions that usually gave the best results (use of acetosyringone before and during the co-cultivation step, $\mathrm{pH} 5.5$ in all fungal media, cocultivation at $23^{\circ} \mathrm{C}$ for $48 \mathrm{~h}$ ). Moreover, in a preliminary study, we compared the two commonly used A. tumefaciens strains EHA101 and AGL1. The results prompted us to select EHA101 for a more efficient ATMT (data not shown). Two other important parameters (mycelium ageing and melanin content) that may modulate ATMT efficiency and have received little attention in the literature were tested in the present study.

Firstly, we hypothesized that the ageing of the initial fungal material could affect ATMT efficiency. In the literature, only two studies have investigated this topic with nonDSE species (Chen et al. 2009; Li et al. 2005). Li et al (2005) reported that ATMT efficiency in Coniothyrium minitans increased with conidia maturation. Likewise, Chen et al (2009) observed that 25-d-old mycelium produced more transformants of Antrodia cinnamomea than 10-d-old mycelium. These observations are in agreement with part of our results. Indeed, 15d-old Leptodontidium sp. Me07, Leptodontidium sp. Pr30 and C. cladosporioides CBS 101367 mycelium was more efficiently transformed than 2-d-old mycelium. However, $P$. mustea Pr29 yielded opposite results. Thick cell walls or cell melanin might prevent T-DNA from entering the host genome (Liu et al. 2010; Michielse et al. 2004; Tsuji et al. 2003). Other mechanisms related to mycelium ageing (such as enzymatic activity, apoptosis, senescence, or cell wall integrity...) could also influence ATMT efficiency for some strains.

Secondly, we also evaluated the influence of mycelial melanin content on ATMT efficiency. The DSEs displayed a diversity of colored mycelia due to different melanin content. Melanin was isolated from the different mycelia, purified, and quantified by spectrophotometry. To our knowledge, this is the first report comparing the melanin content of DSEs from different species and genera. Mycelial melanin content ranged between 0.8 and $3.2 \mathrm{mg} \mathrm{g}^{-1}$ of mycelium. Surprisingly, the red DSE P. mustea Va46 had the highest mycelial melanin content as compared to black and brown fungi. This result could be explained by the elevated concentration of red or yellow melanin, the so-called pheomelanin which absorbs at 
the same wavelength as brown and black melanins (DHN- or DOPA- melanins) (Gessler et al. 2014).

We further studied the relationship between mycelial melanin content and ATMT efficiency. However, no correlation was found, suggesting that ATMT efficiency was not affected by the degree of melanization of the different DSEs. To verify this finding, a biochemical approach was used to modulate the melanin content of four DSEs, and colonies were tested for ATMT. According to Zhan et al. (2011) and Wheeler (1983), DHN-melanin, synthesized from 1,3,6,8-tetrahydroxynaphtalene, is admittedly the main form of melanin produced by ascomycetes. These conclusions prompted us to use tricyclazole as an inhibitor of melanin synthesis. Tricyclazole at non-toxic concentrations is indeed reported to be the strongest systemic fungicide used to specifically block the synthesis of DHN-melanin (Bell \& Wheeler, 1986). This treatment leads to a change in fungal pigmentation (red/brown) as a result of the accumulation of flaviolin and 2-hydroxyjuglone, two major auto-oxidation products of DHN intermediates (Romero-Martinez et al. 2000). In response to tricyclazole, the melanin content of all DSEs significantly decreased. As far as DSEs are concerned, a similar finding was first reported with E. pisciphila, but no quantification was performed and the result was solely based on visual observations (Zhan et al. 2011). Concerning the copper treatment, it promoted melanin synthesis. This $\mathrm{Cu}$-mediated increase of cell wall pigmentation was previously observed in several fungi such as Suillus sp., Amorphotheca resinae, Aureobasidium pullulans, and Gaeumannomyces graminis (Fogarty \& Tobin, 1996; Gadd \& Griffiths, 1980). Copper is known to induce laccases and other enzymes involved in the melanin biosynthesis pathway that could promote hyphal melanization (Eisenman $\&$ Casadevall, 2012). Neither of these two parameters (copper and tricyclazole) had a significant effect on ATMT efficiency. Melanin is composed of individual phenolic components connected together by randomly formed bonds. Melanin can increase resistance against various environmental stresses such as oxidant conditions (ROS), drought, UV and pathogen attacks (Bloomfield \& Alexander, 1967). However, our data show that the T-DNA transfer from $A$. tumefaciens to hyphae was not affected by mycelial melanin content. Therefore our results do not support the previous hypothesis of Liu et al. (2010). However, melanin synthesis might not be totally inhibited in tricyclazole-treated mycelium. Other forms of melanin might still have been present in hyphae, although in minor quantities. The quantification method did not allow us to discriminate between the different forms of melanin. Therefore, we also used sulcotrione and kojic acid to specifically inhibit pyomelanin 
and DOPA-melanin, respectively. Under these conditions, DSE pigmentation was not affected (unpublished results), confirming that DHN-melanin is the major form of melanin in the species we studied. Some of the red-brown pigmented intermediates found under tricyclazole treatment may have increased absorbance. However, more experiments are needed to definitively ascertain that melanin does not impede ATMT of fungi.

\section{Conclusion}

This article reports the first comparison of ATMT efficiency in DSEs, based on eight strains belonging to different DSE species. ATMT efficiency was not affected by mycelial melanin content but was strain-dependent. Within a same species, strain response could vary from very low to high susceptibility to ATMT. Ideally, and in the perspectives of functional post-genomics studies, future sequencing programs of fungal genomes should preferentially select ATMT-susceptible strains to be sequenced. Older mycelium from three DSEs was significantly more susceptible to A. tumefaciens transformation than young mycelium. This point deserves further experiments to investigate the mechanisms that favor age-dependent ATMT efficiency. Based on the present findings, we established an efficient ATMT system for DSEs and we selected Leptodondidium sp. Me07 as a model for future functional genomic studies. To this purpose, we are currently generating a mutant library that will be further screened for different traits of interest.

\section{Acknowledgements}

We would like to thank Christine Friry, Audrey Martin-Mériadec, and Aurianne Aubry for technical support in melanin extraction and fungal transformation. This study was supported by the LORVER project funded by the Lorraine Region and the European Regional Development Fund (ERDF). It was also supported by the French ANR Research Program [grant number ANR BIOFILTREE 2010-INTB-1703-01] and by the Agence de l'Environnement et de la Maitrise de l'Energie (ADEME) [grant number PROLIPHYT 1172C0053].

\section{References}

Addy HD, Piercey MM, Currah RS, 2005. Microfungal endophytes in roots. Canadian Journal of Botany 83:1-13.

Alberton O, Kuyper TW, Summerbell RC, 2009. Dark septate root endophytic fungi increase 
growth of Scots pine seedlings under elevated $\mathrm{CO}_{2}$ through enhanced nitrogen use efficiency. Plant and Soil 328:459-470.

Ban Y, Tang M, Chen H, Xu Z, Zhang H, Yang Y, 2012. The response of dark septate endophytes (DSE) to heavy metals in pure culture. PLoS One 7:e47968.

Barrow JR, 2003. Atypical morphology of dark septate fungal root endophytes of Bouteloua in arid southwestern USA rangelands. Mycorrhiza 13:239-247.

Bell AA, Wheeler MH, 1986. Biosynthesis and functions of fungal melanins. Annual Review of Phytopathology 24:411-451.

Bellion M, Courbot M, Jacob C, Guinet F, Blaudez D, Chalot M, 2007. Metal induction of a Paxillus involutus metallothionein and its heterologous expression in Hebeloma cylindrosporum. New Phytologist 174: 151-8.

Berthelot C, Leyval C, Foulon J, Chalot M, Blaudez D, 2016. Plant growth promotion, metabolite production and metal tolerance of dark septate endophytes isolated from metal-polluted poplar phytomanagement sites. FEMS Microbiology Ecology 92: fiw144

Bloomfield BJ, Alexander M, 1967. Melanins and resistance of fungi to lysis. Journal of Bacteriology 93:1276-1280.

Butler M, Day A, 1998. Fungal melanins: a review. Canadian Journal of Microbiology 44:1115-1136.

Cardoso PG, De Queiroz MV, Pereira OL, De Araújo EF, 2007. Morphological and molecular differentiation of the pectinase producing fungi Penicillium expansum and Penicillium griseoroseum. Brazilian Journal of Microbiology 38:71-77.

Chen ECF, Su YH, Kanagarajan S, Agrawal DC, Tsay HS, 2009. Development of an activation tagging system for the basidiomycetous medicinal fungus Antrodia cinnamomea. Mycological Research $113: 290-297$.

Combier J, Melayah D, Raffier C, Gay G, Marmeisse R, 2003. Agrobacterium tumefaciensmediated transformation as a tool for insertional mutagenesis in the symbiotic ectomycorrhizal fungus Hebeloma cylindrosporum. FEMS Microbiology Letters 220:141-148.

David AS, Haridas S, Labutti K, Lim J, Lipzen A, Wang M, Barry K, Grigoriev IV, Spatafora JW, 2016. Draft genome sequence of Microdochium bolleyi, a dark septate fungal endophyte of beach grass. American society for microbiology 4:1-2.

Deram A, Languereau F, Van Haluwyn C, 2011. Mycorrhizal and endophytic fungal colonization in Arrhenatherum elatius L. roots according to the soil contamination in heavy metals. Soil and Sediment Contamination. $20: 114-127$.

Eisenman HC, Casadevall A, 2012. Synthesis and assembly of fungal melanin. Applied Microbiology and Biotechnology 93:931-940.

Fitzgerald A, Mudge A, Gleave A, Plummer K, 2003. Agrobacterium and PEG-mediated transformation of the phytopathogen Venturia inaequalis. Mycological Research 107;803-810.

Flowers JL, Vaillancourt LJ, 2005. Parameters affecting the efficiency of Agrobacterium tumefaciens-mediated transformation of Colletotrichum graminicola. Current Genetics 48:380-388.

Fogarty RV, Tobin JM, 1996. Fungal melanins and their interaction with metals. Enzyme and 
Microbial Technology 19:311-317.

Gadd GM, Griffiths AJ, 1980. Effect of copper on morphology of Aureobasidium pullulans. Transactions of the British Mycological Society 74:387-392.

Gessler NN, Egorova AS, Belozerskaya TA, 2014. Melanin pigments of fungi under extreme environmental conditions (Review). Applied Biochemistry and Microbiology 50:105113.

Gorfer M, Klaubauf S, Bandian D, Strauss J, 2007. Cadophora finlandia and Phialocephala fortinii: Agrobacterium-mediated transformation and functional GFP expression. Mycological Research 111:850-855.

Gorfer M, Persak H, Berger H, Brynda S, Bandian D, Strauss J, 2009. Identification of heavy metal regulated genes from the root associated ascomycete Cadophora finlandica using a genomic microarray. Mycological Research 113:1377-1388.

Herrera-estrella A, Olmedo-monfil V, Cortés-penagos C, 2004. Three decades of fungal transformation : key concepts and applications, in: Walker J.M. (eds) Methods in Molecular Biology. Springer. pp. 297-313.

Jiang D, Zhu W, Wang Y, Sun C, Zhang K-Q, Yang J, 2013. Molecular tools for functional genomics in filamentous fungi: recent advances and new strategies. Biotechnology Advances 31:1562-1574.

Jumpponen A, Trappe JM, 1998. Dark-septate root endophytes: A review with special reference to facultative biotrophic symbiosis. New Phytologist 140:295-310.

Khastini RO, Ogawara T, Sato Y, Narisawa K, 2014. Control of Fusarium wilt in melon by the fungal endophyte, Cadophora sp. European Jouranl of Plant Pathology 139:333342.

Knapp DG, Pintye A, Kovács GM, 2012. The dark side is not fastidious-dark septate endophytic fungi of native and invasive plants of semiarid sandy areas. PLoS One 7:e32570.

Li M, Gong X, Zheng J, Jiang D, Fu Y, Hou M, 2005. Transformation of Coniothyrium minitans, a parasite of Sclerotinia sclerotiorum, with Agrobacterium tumefaciens. FEMS Microbiology Letters 243:323-9.

Likar M, Regvar M, 2013. Isolates of dark septate endophytes reduce metal uptake and improve physiology of Salix caprea L.. Plant and Soil 370:593-604.

Liu T, Liu L, Jiang X, Hou J, Fu K, Zhou F, Chen J, 2010. Agrobacterium-mediated transformation as a useful tool for the molecular genetic study of the phytopathogen Curvularia lunata. European Journal of Plant Pathology 126:363-371.

Mandyam KG, Jumpponen A, 2005. Seeking the elusive function of the root-colonising dark septate endophytic fungi. Studies in Mycology 53:173-189.

Mandyam KG, Jumpponen A, 2015. Mutualism parasitism paradigm synthesized from results of root-endophyte models. Frontier in Microbiology 5:1-13.

Mandyam KG, Jumpponen A, 2014. Unraveling the dark septate endophyte functions: insights from the Arabidospis model, in: Verma, V.C., Gange, A.C. (Eds.), Advances in Endophytic Research. Springer India. pp. 115-141.

Mayerhofer MS, Kernaghan G, Harper KA, 2013. The effects of fungal root endophytes on plant growth: A meta-analysis. Mycorrhiza 23:119-128. 
Meng Y, Patel G, Heist M, Betts MF, Tucker SL, Galadima N, Donofrio NM, Brown D, Mitchell TK, Li L, Xu J-R, Orbach M, Thon M, Dean RA, Farman ML, 2007. A systematic analysis of T-DNA insertion events in Magnaporthe oryzae. Fungal Genetic and Biology 44:1050-64.

Meyer V, 2008. Genetic engineering of filamentous fungi-progress, obstacles and future trends. Biotechnology Advances 26:177-185.

Michielse CB, Hooykaas PJJ, van den Hondel CAMJJ, Ram AFJ, 2005. Agrobacteriummediated transformation as a tool for functional genomics in fungi. Current Genetics 48:1-17.

Michielse CB, Salim K, Ragas P, Ram AFJ, Kudla B, Jarry B, Punt PJ, van den Hondel CAMJJ, 2004. Development of a system for integrative and stable transformation of the zygomycete Rhizopus oryzae by Agrobacterium-mediated DNA transfer. Molecular Genetics and Genomics 271:499-510.

Mullins ED, Kang S, 2001. Transformation: a tool for studying fungal pathogens of plants. Cellular and Molecular Life Science 58:2043-2052.

Munsell AH, 1954. Munsell soil color chart, in: Munsell Color CO. Baltimore, Maryland, U.S.A.

Newsham KK, 2011. A meta-analysis of plant responses to dark septate root endophytes. New Phytologist 190:783-93.

Pardo AG, Hanif M, Raudaskoski M, Gorfer M, 2002. Genetic transformation of ectomycorrhizal fungi mediated by Agrobacterium tumefaciens. Mycological Research 106:132-137.

Romero-Martinez R, Wheeler MH, Guerrero-Plata A, Rico G, Torres-Guerrero H, 2000. Biosynthesis and function of melanin in Sprorothrix schenckii. Infection and Immunity 68:3696-3703.

Saitou N, Nei M, 1987. The neighbor-joining method - a new method for reconstructing phylogenetic trees. Molecular Biology and Evolution 4:406-425.

Sonjak S, Udovič M, Wraber T, Likar M, Regvar M, 2009. Diversity of halophytes and identification of arbuscular mycorrhizal fungi colonising their roots in an abandoned and sustained part of Sečovlje salterns. Soil Biology and Biochemistry 41:1847-1856.

Su Z-Z, Mao L-J, Li N, Feng X-X, Yuan Z-L, Wang L-W, Lin F-C, Zhang C-L, 2013. Evidence for biotrophic lifestyle and biocontrol potential of dark septate endophyte Harpophora oryzae to rice blast disease. PLoS One 8:e61332.

Tamura K, Stecher G, Peterson D, Filipski A, Kumar S, 2013. MEGA6: Molecular evolutionary genetics analysis version 6.0. Molecular Biology and Evolution 30:2725-9.

Tellenbach C, Sumarah MW, Grünig CR, Miller JD, 2013. Inhibition of Phytophthora species by secondary metabolites produced by the dark septate endophyte Phialocephala europaea. Fungal Ecology 6:12-18.

Tsuji G, Fujii S, Fujihara N, Hirose C, Tsuge S, Shiraishi T, Kubo Y, 2003. Agrobacterium tumefaciens-mediated transformation for random insertional mutagenesis in Colletotrichum lagenarium. Journal of General Plant Pathology 69:230-239.

Tzima AK, Paplomatas EJ, Schoina C, Domazakis E, Kang S, Goodwin PH, 2014. Successful Agrobacterium mediated transformation of Thielaviopsis basicola by optimizing multiple conditions. Fungal Biology 118:675-682. 
Upson R, Read DJ, Newsham KK, 2009. Nitrogen form influences the response of Deschampsia antarctica to dark septate root endophytes. Mycorrhiza 20:1-11.

Usuki F, Narisawa K, 2007. A mutualistic symbiosis between a dark septate endophytic fungus, Heteroconium chaetospira, and a nonmycorrhizal plant, Chinese cabbage. Mycologia 99:175-84.

Vági P, Knapp DG, Kósa A, Seress D, Horváth ÁN, Kovács GM, 2014. Simultaneous specific in planta visualization of root-colonizing fungi using fluorescence in situ hybridization (FISH). Mycorrhiza 24:259-266.

Van Duin D, Casadevall A, Nosanchuk JD, 2002. Melanization of Cryptococcus neoformans and Histoplasma capsulatum reduces their susceptibilities to amphotericin B and caspofungin. Antimicrobial Agents and Chemotherapy 46:3394-3400.

Vellinga EC, 2006. Lepiotaceous fungi in California, U.S.A. -2 Lepiota rhodophylla sp.nov. Mycotaxon 98:205-211.

Vrålstad T, 2002. Molecular diversity and phylogenetic affinities of symbiotic root-associated ascomycetes of the Helotiales in burnt and metal polluted habitats. New Phytologist 153:131-148.

Wang C, Gua, X, Wang H, Li G, Dong X, Wang G, Li B, 2013. Agrobacterium tumefaciensmediated transformation of Valsa mali: An efficient tool for random insertion mutagenesis. The Scientific World Journal 2013:1-11.

Wheeler BMH, 1983. Comparisons of fungal melanin biosynthesis in ascomycetous, imperfect and basidiomycetous fungi. Transactions of the British Mycological Society 81:29-36.

White T, Bruns T, Lee S, et al., 1990. Amplification and direct sequencing of fungal ribosomal RNa genes for phylogenetics, in: Innis, M.A., Gelfand, D.H., Sninsky, J.J., White, T.J. (Eds.), PCR-protocol and applications - a laboratory manual. Academic Press New York. pp. 315-322.

Xu X-H, Su Z-Z, Wang C, Kubicek CP, Feng X-X, Mao L-J, Wang J-Y, Chen, C, Lin F-C, Zhang C-L, 2014. The rice endophyte Harpophora oryzae genome reveals evolution from a pathogen to a mutualistic endophyte. Scientific Reports 4, 5783.

Zhan F, He Y, Zu Y, Li T, Zhao Z, 2011. Characterization of melanin isolated from a dark septate endophyte (DSE), Exophiala pisciphila. World Journal of Microbiology and Biotechnology 27: 2483-2489.

Zhang P, Liu TT, Zhou PP, Li ST, Yu LJ, 2011. Agrobacterium tumefaciens-mediated transformation of a taxol-producing endophytic fungus, Cladosporium cladosporioides MD2. Current Microbiology 62:1315-1320.

Zhang Y, Li T, Zhao Z-W, 2013. Colonization characteristics and composition of dark septate endophytes (DSE) in a lead and zinc slag heap in southwest China. Soil and Sediment Contamination: An International journal J. 22:532-545.

Zhao D, Li T, Wang J, Zhao Z, 2015. Diverse strategies conferring extreme cadmium (Cd) tolerance in the dark septate endophyte (DSE), Exophiala pisciphila: Evidence from RNA-seq data. Microbiological Research 170:27-35.

Zou Y, Xie C, Fan G, Gu Z, Han Y, 2010. Optimization of ultrasound-assisted extraction of melanin from Auricularia auricula fruit bodies. Innovative Food Science and Emerging Technologies 11:611-615. 